\title{
Gamma-ray emission from massive star forming regions
}

\author{
A. T. ARAUDO* and G. E. ROMERO** \\ Instituto Argentino de Radioastronomía, C.C.5, (1894) Villa Elisa, Buenos Aires, Argentina \\ Facultad de Cs. Astronómicas y Geofísicas, Universidad Nacional de La Plata, Paseo del Bosque, 1900 La Plata, Argentina \\ *E-mail: aaraudo@fcaglp.unlp.edu.ar \\ **E-mail: romero@fcaglp.unlp.edu.ar \\ V. BOSCH-RAMON \\ Max Planck Institut für Kernphysik, Saupfercheckweg 1, Heidelberg 69117, Germany \\ E-mail:Valenti.Bosch-Ramon@mpi-hd.mpg.de \\ J. M. PAREDES \\ Departament d'Astronomia i Meteorologia, Universitat de Barcelona, Martí i Franquès 1, 08028, Barcelona, Spain \\ E-mail: jmparedes@ub.edu
}

\begin{abstract}
Recent radio observations support a picture for star formation where there is accretion of matter onto a central protostar with the ejection of molecular outflows that can affect the surrounding medium. The impact of a supersonic outflow on the ambient gas can produce a strong shock that could accelerate particles up to relativistic energies. A strong evidence of this has been the detection of non-thermal radio emission coming from the jet termination region of some young massive stars. In the present contribution, we study the possible high-energy emission due to the interaction of relativistic particles, electrons and protons, with the magnetic, photon and matter fields inside a giant molecular cloud. Electrons lose energy via relativistic Bremsstrahlung, synchrotron radiation and inverse Compton interactions, and protons cool mainly through inelastic collisions with atoms in the cloud. We conclude that some massive young stellar objects might be detectable at gamma-rays by next generation instruments, both satellite-borne and ground based.
\end{abstract}

Keywords: Gamma-ray emission; Massive stars: formation; Radiation mechanisms: non-thermal

\section{Introduction}

The mechanism of formation of massive stars $(M>$ $\left.8 M_{\odot}\right)$ remains one of the open questions in astrophysics. It is known that these stars originate inside giant and massive molecular clouds but the sequence of processes that take place during the formation of the star are mostly unknown. It has been suggested, for example, that the coalescence of various protostars in the same cloud can lead to the emergence of a massive star [1]. Massive stars appear in massive stellar associations where cloud fragmentation seems to be common. Alternatively, a massive star could form by the collapse of the core of a molecular cloud, with associated episodes of mass accretion and ejection, as observed in low-mass stars [2]. In such a case, the effects of jets propagating through the medium that surrounds the protostar should be detectable.

Until now, the formation of stars has been mostly associated with thermal radio and X-ray emission. However, non-thermal radio emission has been detected in some massive star forming regions. This is a clear evidence that efficient particle acceleration is occurring there, which may have as well a radiative outcome at energies much higher than radio ones.

In the present contribution, and based on recent multiwavelength observations, as well as reasonable physical assumptions, we show that massive protostars could produce a significant amount of radiation in the gamma-ray domain, because of the dense and rich medium in which they are formed.

\section{Non-thermal radio sources}

In the last years, synchrotron radiation have been observed from some regions where massive stars form. This emission is associated with outflows emanating from a central protostar. In what follows, we briefly describe some of these non-thermal sources that could be potential emitters of gamma-rays.

\subsection{IRAS 16547-4247}

The triple radio source associated with the protostar IRAS $16547-4247$ is one of the best candidates to 
produce gamma-rays. This system is located within a very dense region (i.e. densities $n \approx 5 \times 10^{5} \mathrm{~cm}^{-3}$ ) of a giant molecular cloud located at a distance of $2.9 \mathrm{kpc}$. The luminosity of the IRAS source is $L=6.2 \times 10^{4} L_{\odot}$, possibly being the most luminous known YSO associated with collimated thermal jets.

ATCA and VLA observations [3, 4] have shown that the southern lobe of this system, of size $\sim$ $10^{16} \mathrm{~cm}$, has a clear non-thermal spectrum, with an index $\alpha \sim 0.6\left(S_{\nu} \propto \nu^{-\alpha}\right)$. The specific flux of this source is $2 \mathrm{mJy}$ at $14.9 \mathrm{GHz}$ and the estimated magnetic field is $B \sim 2 \times 10^{-3} \mathrm{G}$ [5].

\subsection{Serpens}

The Serpens molecular cloud is located at a distance of $\sim 300$ pc. One of the two central dense cores of this cloud is a triple radio source, composed by a central protostar (IRAS 18273-0113) and two lobes. The northwest (NW) hot-spot is connected with the central source by a highly collimated thermal jet, whereas the southeasth (SE) is separated and broken into two clumps. The luminosity of the source IRAS $18273-0113$ is $L \sim 300 L_{\odot}$ and the particle density at the center of the molecular cloud is $n_{0} \sim 10^{5} \mathrm{~cm}^{-3}$.

The observed radio emission $[6,7]$ detected from the central and NW sources has a spectral index $\alpha \approx-0.15$ and $\alpha \approx 0.05$, respectively. This emission, of a luminosity $\sim 2-3 \mathrm{mJy}$, is produced via thermal Bremsstrahlung. However, the radiation produced in the SE lobe seems to be non-thermal ( $\alpha=0.3)$, likely produced via synchrotron emission. The specific flux of this source is $2-5 \mathrm{mJy}$. The equipartition magnetic field estimated in the SE lobe is $B_{\text {equip }} \sim 10^{-3} \mathrm{G}[6]$.

\subsection{HH 80-81}

The famous Herbig-Haro objects called HH 80-81 are the south component of a system of radio sources, located in the Sagitarius region, at a distance of $1.7 \mathrm{kpc}$. The central source has been identified with the luminous $\left(L=1.7 \times 10^{4} L_{\odot}\right)$ protostar IRAS 18162-2048. HH 80 North is the northern counterpart of $\mathrm{HH} 80-81$. The velocity of the jet has been estimated as $v \sim 700 \mathrm{~km} \mathrm{~s}^{-1}$, allowing to derive a dynamical age for the system similar to $4000 \mathrm{yr}$.

Radio observations carried out with the VLA instrument [8] showed that the central source has a spectral index $\alpha \sim 0.1$, typical of free-free emission, whereas HH 80-81 and HH 80 North are likely nonthermal sources, with spectral index $\alpha \sim 0.3$. The specific flux measured at a frecuency of $5 \mathrm{GHz}$ is $F_{\nu} \sim 1-2 \mathrm{mJy}$ and $F_{\nu} \approx 2.4 \mathrm{mJy}$ for the sources $\mathrm{HH}$ 80-81 and HH 80 North, respectively. At this frecuency, the angular size of the north and the south components are $\sim 6^{\prime \prime}$.

In addition, $\mathrm{HH} 80-81$ system is a powerful source of thermal X-rays with a luminosity $L_{X} \sim$ $4.3 \times 10^{31} \mathrm{erg} \mathrm{s}^{-1}[9]$.

\section{4. $W 3(\mathrm{OH})$}

Other interesting source to study is the system composed by an $\mathrm{H}_{2} \mathrm{O}$ maser complex and the TurnerWelch (TW) source in the W3 region [10]. The central source of this system is a luminous $\left(L \sim 10^{5} L_{\odot}\right)$ YSO and the mean density of cool particles is $n \sim$ $4 \times 10^{4} \mathrm{~cm}^{-3}$. The distance to $\mathrm{W} 3(\mathrm{OH})$ is $2.2 \mathrm{kpc}$.

Continuum radio observations [10] show the presence of a sinuous double-sided jet, emanating from the TW source. The observed radio flux, from 1.6 to $15 \mathrm{GHz}$, is in the range $2.5-0.75 \mathrm{mJy}$, and the spectral index of the observed emission is clearly nonthermal: $\alpha=0.6$. The inhomogeneous synchrotron model proposed by Reid et al. in Ref. [11] predicts for the emitting jet a density of relativistic electrons $n_{e}(\gamma, r)=0.068 \gamma^{-2}\left(r / r_{0}\right)^{-1.6} \mathrm{~cm}^{-3}$ and a magnetic field $B(\gamma, r)=0.01\left(r / r_{0}\right)^{-0.8} \mathrm{G}$, where $r_{0}=6.6 \times 10^{15} \mathrm{~cm}$, and $r$ and $\gamma$ are the distance to the jet origin and the electron Lorentz factor, respectively. Unlike in previous cases, here the non-thermal radio emission comes from the jet and not from its termination region. Non-thermal jets associated to a YSO are uncommon.

\section{Acceleration of particles and losses}

The non-thermal radio emission observed in some massive star forming regions is interpreted as synchrotron radiation produced by the interaction of relativistic electrons with the magnetic field present in the cloud, being typically $B_{\text {cloud }} \sim 10^{-3} \mathrm{G}$ [12]. These particles could be accelerated at a shock, formed in the point where the jet terminates, via diffusive shock acceleration [13]. The acceleration efficiency, characterized by $\eta$, is related to the velocity of the shock. Using the values of the velocities given for the sources described in the previous section, and assuming Bohm diffusion, values for $\eta$ of 
$\sim 10^{-6}-10^{-5}$ are obtained.

Particles accelerated up to relativistic energies interact with the different fields present in the medium. As noted at the beginning of this section, electrons radiate synchrotron emission under the ambient magnetic field $B$. In addition, particles, electrons and protons, can also interact with the cold matter in the jet termination region (via relativistic Bremsstrahlung the leptons, and proton-nuclei collisions the protons). In addition, electrons interact with the background field of IR photons of the protostar, of energy density $u_{\mathrm{ph}}$, via inverse Compton (IC) scattering.

Using the following parameter values: $n=5 \times$ $10^{5} \mathrm{~cm}^{-3} ; B=2.5 \times 10^{-3} \mathrm{G}$; and $u_{\mathrm{ph}}=3.2 \times$ $10^{-9} \mathrm{erg} \mathrm{cm}^{-3}$ given for IRAS 16547-4247, [3, 5], we estimate the cooling time of the main leptonic processes in this scenario. As seen in Fig. 1, relativistic Bremsstrahlung losses are dominant up to $\sim 10 \mathrm{GeV}$. In addition, it is possible to see from this figure that the maximum energy achieved by electrons is $E_{e}^{\max } \sim 1 \mathrm{TeV}$ and is determined by synchrotron losses, being IC losses negligible.

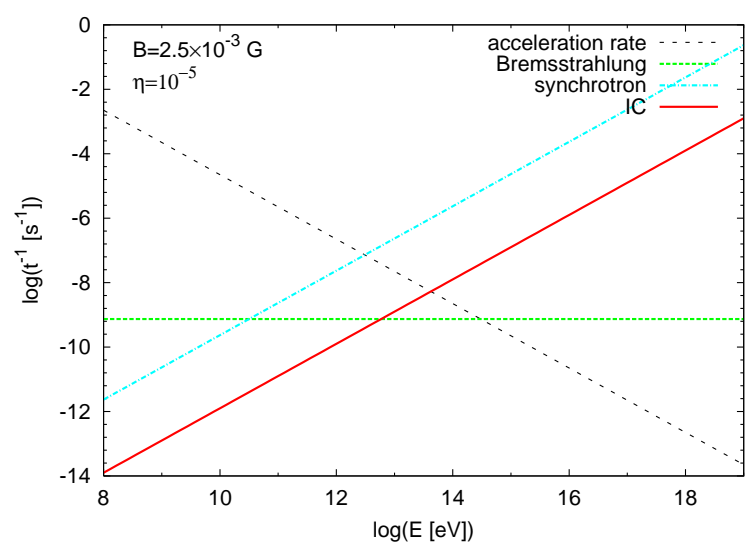

Fig. 1. Energy loss and acceleration rates for electrons in the IRAS $16547-4247$ southern lobe.

Protons can be accelerated by the shock in the same way as electrons and interact with cold particles present in the cloud. The maximum energy achieved by protons is higher: $E_{p}^{\max } \sim 10 \mathrm{TeV}$. In $p p$ interactions, besides $\gamma$-rays, secondary electron-positron pairs are produced. These secondary particles will radiate by the same mechanisms as primary electrons (i.e. synchrotron radiation, IC scattering and relativistic Bremsstrahlung).

\section{High-energy emission}

In order to calculate the non-thermal spectral energy distribution (SED) of a massive protostar, the magnetic field of the cloud and the distributions of relativistic particles, $n(E)$, are needed. To obtain the magnetic field $B$ and the normalization constants of the particle distributions, we use the standard equations given by Ginzburg \& Syrovatskii [14] for the observed synchrotron flux and assuming equipatition between the magnetic and the relativistic particle (primary and secondary pairs and protons) energy densities:

$$
\frac{B^{2}}{8 \pi}=u_{e}+u_{p}+u_{e^{ \pm}},
$$

In Eq. (1), the following relationships are implicit: $u_{\mathrm{p}}=a u_{\mathrm{e}}$ and $u_{e^{ \pm}}=f u_{\mathrm{p}}$. In the first relation $a$ is a free parameter (here fixed to 100 , as it is the case for cosmic rays) and in the second relation $f$ can be estimated using the average ratio of the number of secondary pairs to $\pi^{0}$-decay photons in Ref. [15]. In Fig. 2, we show the computed broadband SED for the parameters of the source IRAS 16547-4247.

\section{Discussion}

In this work we show that, if the source is located at few kpc, the high-energy emission may be detected by GLAST and even by forthcoming Cherenkov telescope arrays after long enough exposure. This opens a new window to the study of star formation and related processes. Also, determinations of the particle spectrum and its high-energy for different sources with a variety of environmental conditions can shed light on the properties of galactic, supersonic outflows, and on the particle acceleration processes occurring at their termination points.

Radio observations already demonstrate that relativistic electrons are produced in some sources. According to the presence of non-thermal emission detected at cm-wavelengths and IR observations of the protostars emission we can suggest several good candidates to be targeted by GLAST. These objects are IRAS 16547-4247 [5], HH 80-81 [8], W3 [10, 11] and the multiple radio source in Serpens $[6,7]$

To conclude, we emphasize that massive YSO with bipolar outflows and non-thermal radio emission can form a new population of gamma-ray sources that could be unveiled by the next generation of $\gamma$-ray instrumentation. 


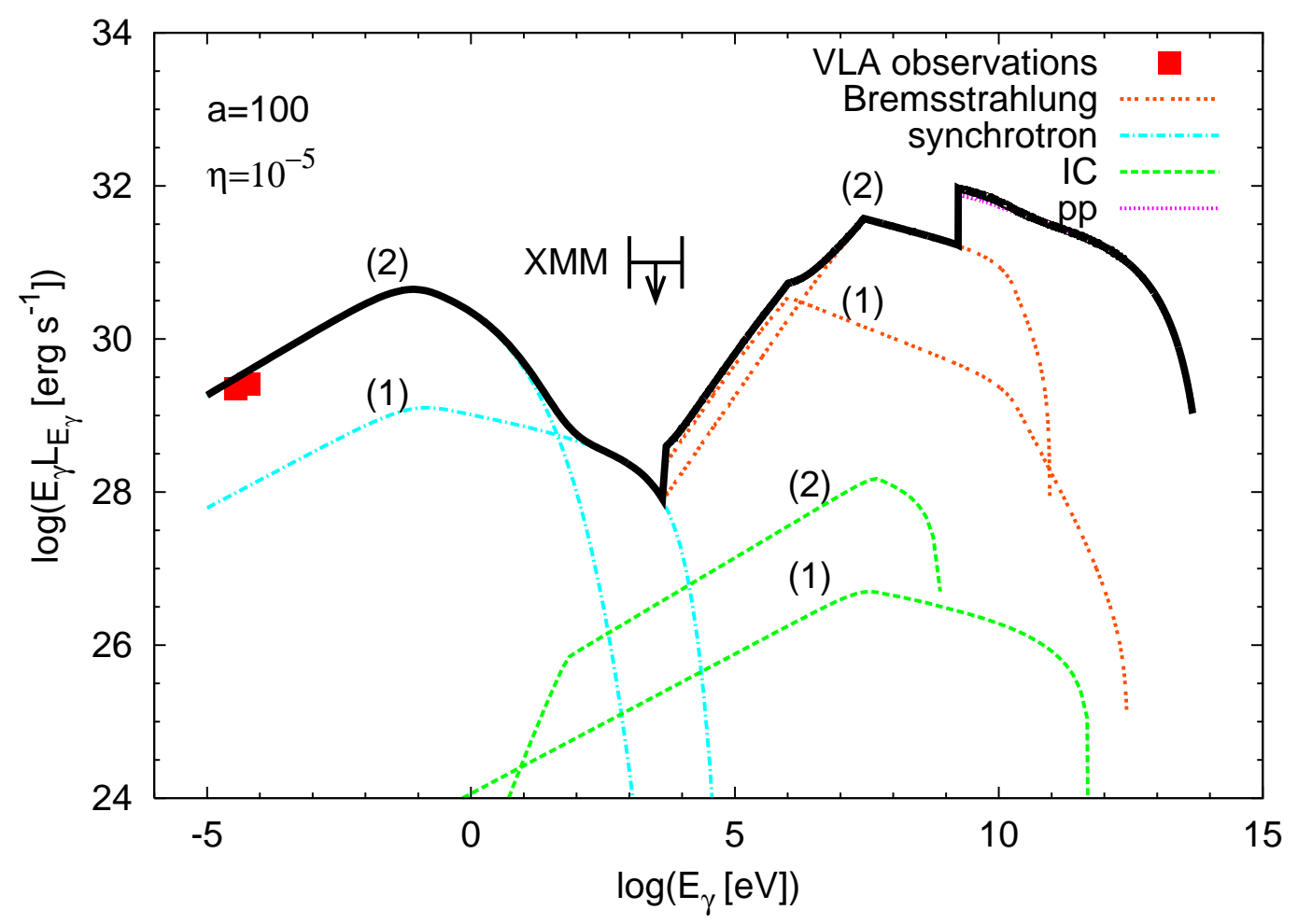

Fig. 2. Spectral energy distribution for the south lobe of the YSO embedded in the source IRAS 16547-4247. The radiative contribution of secondary pairs (2) produced via $\pi^{ \pm}$-decay is shown along with the contribution of primary electrons (1).

\section{Acknowledgements}

A.T.A. \& G.E.R. are supported by the Argentine Agencies CONICET (GRANT PIP 5375) and ANPCyT (GRANT PICT 03-13291 BID 1728/OC-AR). V.B-R., and J.M.P acknowledge support by DGI of MEC under grant AYA2007-6803407171-C03-01, as well as partial support by the European Regional Development Fund (ERDF/FEDER). V.B-R. gratefully acknowledges support from the Alexander von Humboldt Foundation.

\section{References}

[1] I. A. Bonnell, M. R. Bate \& H. Zinnecker MNRAS 298, 93 (1998).

[2] F. H. Shu, F. C. Adams \& S. Lizano ARA\& A 25, 23 (1987).

[3] G. Garay, K. Brooks, D. Mardones \& R. P. Norris ApJ 537, 739 (2003).
[4] L. F. Rodriíguez, G. Garay, K. J. Brooks \& D. Mardones ApJ 626, 953 (2005).

[5] A. T. Araudo, G. E. Romero, V. Bosch-Ramon \& J. M. Paredes $A \mathscr{E} A$ A 476, 1289A (2007).

[6] L. F. Rodríguez, S. Curriel, J. M. Morán, I. F. Mirabel, M. Roth \& G. Garay ApJ 346, L85 (1989).

[7] S. Curriel, L. F. Rodríguez, J. M. Morán \& J. Cantó ApJ 415, 191 (1993).

[8] J. Martí, L. F. Rodríguez, \& B. Reipurth ApJ 449, 184 (1993).

[9] S. H. Pravdo, Y. Tsuboi \& Y. Maeda ApJ 605, 259 (2004).

[10] D. J. Wilner, M. J. Reid, \& K. M. Menten $A p J$ 513, 775 (1999).

[11] M. J. Reid, A. L. Argon, K. M. Menten, \& J. M. Moran ApJ 443, 238 (1995).

[12] R. M. Crutcher $A p J$ 520, 706 (1999).

[13] L. O'C. Drury Rep. Prog. Phys. 46, 973 (1983).

[14] V. L. Ginzburg \& S. I. Syrovatskii The Origin of Cosmic Rays, (Pergamon Press, New York, 1964).

[15] S. R. Kelner, F. A. Aharonian, \& V. V. Vugayov Phys. Rev. D 74, 034018 (2006). 